\title{
Human liver mesenchymal stem/progenitor cells inhibit hepatic stellate cell activation: in vitro and in vivo evaluation
}

\author{
Mustapha Najimi ${ }^{1 *} \mathbb{D}$, Silvia Berardis ${ }^{1+}$, Hoda El-Kehdy $^{1+}$, Valérie Rosseels $^{1+}$, Jonathan Evraerts ${ }^{1}$, \\ Catherine Lombard ${ }^{1}$, Adil El Taghdouini ${ }^{1}$, Patrick Henriet ${ }^{3}$, Leo van Grunsven ${ }^{2}$ and Etienne Marc Sokal ${ }^{1}$
}

\begin{abstract}
Background: Progressive liver fibrosis leads to cirrhosis and end-stage liver disease. This disease is a consequence of strong interactions between matrix-producing hepatic stellate cells (HSCs) and resident and infiltrating immune cell populations. Accumulated experimental evidence supports the involvement of adult-derived human liver mesenchymal stem/progenitor cells (ADHLSCs) in liver regeneration. The aim of the present study was to evaluate the influence of ADHLSCS on HSCs, both in vitro and in vivo.

Methods: Activated human HSCs were co-cultured with ADHLSCS or ADHLSC-conditioned culture medium. The characteristics of the activated human HSCs were assessed by microscopy and biochemical assays, whereas proliferation was analyzed using flow cytometry and immunocytochemistry. The secretion profile of activated HSCs was evaluated by ELISA and Luminex. ADHLSCs were transplanted into a juvenile rat model of fibrosis established after co-administration of phenobarbital and $\mathrm{CCl}_{4}$.

Results: When co-cultured with ADHLSCs or conditioned medium, the proliferation of HSCs was inhibited, beginning at $24 \mathrm{~h}$ and for up to 7 days. The HSCs were blocked in G0/G1 phase, and showed decreased Ki-67 positivity. Pro-collagen I production was reduced, while secretion of HGF, IL-6, MMP1, and MMP2 was enhanced. Neutralization of HGF partially blocked the inhibitory effect of ADHLSCs on the proliferation and secretion profile of HSCs. Repeated intrahepatic transplantation of cryopreserved/thawed ADHLSCs without immunosuppression inhibited the expression of markers of liver fibrosis in 6 out of 11 rats, as compared to their expression in the vehicle-transplanted group.
\end{abstract}

Conclusions: These data provide evidence for a direct inhibitory effect of ADHLSCs on activated HSCs, which supports their development for the treatment of liver fibrosis.

Keywords: Liver, Liver fibrosis, Liver stem/progenitor cells, Hepatic stellate cells, Secretome

\section{Background}

Control of liver fibrosis is an unmet medical need. Fibrosis occurs in response to chronic liver injury, and if uncontrolled, leads to cirrhosis and end-stage liver disease [1,2]. Patients with liver fibrosis require liver transplantation and face long waiting times with progressive disability [3].

\footnotetext{
* Correspondence: Mustapha.najimi@uclouvain.be

${ }^{\dagger}$ Equal contributors

'Université Catholique de Louvain, Institut de Recherche Expérimentale et Clinique (IREC), Laboratory of Pediatric Hepatology and Cell Therapy, Avenue Mounier, 52, 1200 Brussels, Belgium

Full list of author information is available at the end of the article
}

Even after successful transplant, advanced fibrosis of the graft can occur [4]. Despite an improved understanding of the mechanisms underlying the development of liver fibrosis, the efficacy of most drugs has not yet been proven in humans $[1,5,6]$.

Hepatic stellate cells (HSCs) are the main extracellular matrix-producing cells in the liver. Following injury, HSCs undergo an activation process characterized by the adoption of proliferative, contractile, and fibrogenic myofibroblastic features [7], a process that can be reproduced in plastic culture dishes [8]. The ideal anti-fibrotic therapy should modulate HSC activation, inhibit 
collagen synthesis, and enhance matrix degradation [9]. Mesenchymal stem cells (MSCs) have been proposed for the treatment of fibrosis based on their hepatocyte differentiation and regeneration potential as well as their immunomodulatory properties [10-13]. Adult-derived human liver stem cells (ADHLSCs) are a subtype of MSCs, which can be obtained by collagenase digestion of the liver, with a preferential hepatocyte differentiation pattern $[14,15]$. Like other MSCs, ADHLSCs can produce trophic growth factors and cytokines that are able to overwhelm inflammatory responses $[16,17]$. Moreover, MSCs have the potential to exert a suppressive effect on immune cells $[18,19]$. ADHLSCs are currently in clinical development, and have been safely and successfully infused in children with inborn errors of liver metabolism [11, 12, 20].

Extrahepatic MSCs were reported to reverse fibrosis in animal models of induced liver fibrosis [21, 22], with frequent improvement of hepatic function. However, the underlying mechanisms are not yet well understood. Therefore, in vitro models and further experimentation are required to better understand how MSCs modulate HSC activation and their paracrine properties [23-25]. Ideally, these systems should use humanderived cells since rodent cells may behave and react differently.

In the current study, we investigated the ability of ADHLSCs to modulate the activation of HSCs both in vitro and in vivo. We demonstrated that ADHLSCs efficiently inhibited HSC proliferation and collagen secretion in vitro, mainly via HGF. Increased secretion of other anti-fibrotic factors by treated HSCs was also noted. In vivo, we showed that repeated intrahepatic transplantation of ADHLSCs was correlated with a decrease in the expression of markers related to liver fibrosis. Thus, our data support the further development of ADHLSCs for the treatment of liver fibrosis.

\section{Methods}

\section{ADHLSC and HSC isolation and culture}

This study was approved by the ethics committee of our institution. Liver tissue was obtained from a Ministry of Health-approved hospital tissue bank (Table 1). ADHLSCs were obtained after primary culture of the parenchymal fraction cells, as previously described [14, 17]. The supernatant, containing the non-parenchymal cell fraction, was processed by Nycodenz gradient
(Myegaard, Oslo, Norway) centrifugation to isolate HSCs [26]. Both cell types were cultured in Dulbecco's modified Eagle's medium (DMEM) containing $4.5 \mathrm{~g} / \mathrm{L}$ glucose (Life Technologies, Carlsbad, CA, USA) supplemented with $10 \%$ fetal calf serum (FCS; Life Technologies) and 1\% penicillin/streptomycin (Life Technologies), at $37{ }^{\circ} \mathrm{C}$ in a humidified atmosphere containing $5 \% \mathrm{CO}_{2}$, as previously described [17]. When the cells reached $80 \%$ confluence, they were detached by treatment with $0.05 \%$ TrypsinEDTA (Life Technologies). The viability of the recovered cells was evaluated using the trypan blue exclusion assay.

\section{Co-culture systems}

To evaluate the interactions between ADHLSCs and activated HSCs, indirect co-culture in Transwells was used. HSCs were seeded in the lower chamber of a six-well plate at a density of $1.0 \times 10^{5}$ cells $/ \mathrm{cm}^{2}$, and the ADHLSCs were placed on the collagen-coated membrane inserts $(24 \mathrm{~mm}$ diameter, $0.4 \mu \mathrm{m}$ pore size; Corning, Inc., Corning, NY, USA), at ADHLSC:HSC ratios of 1:100, 1:10, 1:1, and 0:1. HSCs were collected at 1, 4, and 7 days for analysis. Cell numbers and viability were evaluated using microscopy and the trypan blue exclusion assay, respectively. Viability was also estimated with a sensitive colorimetric assay, the Cell Counting Kit-8 (CCK-8; Sigma-Aldrich, St. Louis, MO, USA) according to the manufacturer's instructions.

\section{Flow cytometry}

For cell cycle analysis, $2.0 \times 10^{5}$ cells were used for each experimental condition. Cell suspensions were washed twice with PBS and fixed with $700 \mu \mathrm{L}$ of cold ethanol for $30 \mathrm{~min}$ on ice. The fixed HSCs were washed with PBS, incubated with $100 \mu \mathrm{g} / \mathrm{mL}$ propidium iodide (Life Technologies), $0.1 \mathrm{mg} / \mathrm{mL}$ RNase (Sigma-Aldrich), and $0.1 \%$ Triton X-100 (Sigma-Aldrich) for $30 \mathrm{~min}$ at $37{ }^{\circ} \mathrm{C}$, and then incubated on ice for $15 \mathrm{~min}$. Apoptosis was assessed using the FITC Annexin V Apoptosis Detection Kit I (BD Pharmingen, San Diego, CA, USA) according to the manufacturer's instructions. Cells treated with $1 \mathrm{mM}$ hydrogen peroxide were used as a positive control. Cells were evaluated with a CANTO II flow cytometer, and the data were analyzed with BD FACSDiva software. The number of cells in different phases of the cell cycle was determined by measuring the area

Table 1 Characteristics of the four liver donors whose samples were used for the isolation of HSCs and ADHLSCs used in the current study

\begin{tabular}{llllll}
\hline Donor number & Age & Gender & Cause of death & Blood group & Ischemia time \\
\hline 89 & 3 days & M & Respiratory & A+ & h \\
93 & 2 years & F & Metabolic disease (liver transplanted) & O+ & h 43 min \\
98 & 7 days & M & Cardio-respiratory arrest & O- & $4 \mathrm{~h} \mathrm{20} \mathrm{min}$ \\
105 & 46 years & F & Trauma & B+ & $9 \mathrm{~h} \mathrm{43} \mathrm{min}$ \\
\hline
\end{tabular}


under the curve using FlowJo software (Tree Star, Inc., Ashland, OR, USA).

\section{Immunocytochemistry}

After $24 \mathrm{~h}$ of incubation with ADHLSC- or HSCconditioned medium in 24-well plates, HSCs were fixed with paraformaldehyde (3.5\%) for $15 \mathrm{~min}$ at room temperature. Immunostaining was performed as previously described [17]. Cells were incubated with a Ki-67 antibody (1:150 dilution; Dako, Glostrup, Denmark) for $1 \mathrm{~h}$. For each experimental condition, four different fields were analyzed, and the number of stained/unstained nuclei (for a total of 2500 nuclei) were counted using ImageJ software (US National Institutes of Health, Bethesda, MD, USA).

\section{ELISA}

After removal of the inserts containing ADHLSCs and aspiration of the medium, recovered HSCs were washed with sterile PBS and incubated in serum-free medium (DMEM containing $4.5 \mathrm{~g} / \mathrm{L}$ glucose [Life Technologies]) for $24 \mathrm{~h}$. Then, the supernatant was collected, and cells were detached for counting and evaluation of viability. Collagen secretion was evaluated using an ELISA kit for procollagen type I C-Peptide (Takara Bio Inc., Shiga, Japan). The absorbance at $450 \mathrm{~nm}$ was measured with a VICTOR X4 Plate Reader (Perkin Elmer, Waltham, MA, USA). The results were randomized according to the number of cells collected.

\section{Western blotting}

Total protein lysates were obtained by disrupting $100 \mathrm{mg}$ of rat liver in RIPA buffer [50 mM Tris-Base, $\mathrm{pH}$ 8.0, $150 \mathrm{mM} \mathrm{NaCl}, 1 \%$ Triton X-100, 0.5\% sodium deoxycholate, and $0.1 \%$ SDS, with a protein inhibitor cocktail without EDTA (Roche, Basel, Switzerland)] using FastPrep lysing matrix A beads (MP Biomedicals, Santa Ana, CA, USA) at $6 \mathrm{~m} / \mathrm{s}$ for $30 \mathrm{~s}$. The protein lysates were incubated on ice for $30 \mathrm{~min}$ prior to sample clarification by centrifugation (30 $\mathrm{min}$ at maximum speed). Then, sample supernatants were collected, and total protein was quantified using the BCA quantification kit (Thermo Fisher Scientific, Waltham, MA, USA).

Western blotting was performed as previously described [27]. Briefly, extract containing $50 \mu \mathrm{g}$ of total protein was mixed with loading buffer [Tris- $\mathrm{HCl}(\mathrm{pH}$ 6.8), glycerol, SDS, DTT, and bromophenol blue], denatured by incubation at $95{ }^{\circ} \mathrm{C}$ for $5 \mathrm{~min}$, and then loaded on a $10 \%$ Trisglycine SDS-PAGE gel for protein separation. The separated proteins were transferred onto PVDF membranes overnight at $4{ }^{\circ} \mathrm{C}$. Membranes were incubated with $5 \%$ BSA blocking solution for $60 \mathrm{~min}$ at room temperature, and then with the primary antibodies (see Table 2) overnight at $4{ }^{\circ} \mathrm{C}$. Then, membranes were
Table 2 Antibodies used for western blotting analysis

\begin{tabular}{llll}
\hline & Reference & Concentration used & Supplier \\
\hline Primary & & & \\
Anti-a-SMA & M0851 & $1 / 250$ & Dako \\
Anti-fibronectin & Ab2413 & $2 \mu \mathrm{g} / \mathrm{ml}$ & Abcam \\
Anti-GAPDH & MAB374 & $1 / 5000$ & Millipore \\
Secondary & & & \\
Donkey anti-rabbit & BTIU20418 & $1 / 10000$ & Biotium \\
Donkey anti-mouse & BTIU20363 & $1 / 10000$ & Biotium \\
\hline
\end{tabular}

thoroughly washed with TBS-T, and incubated with fluorescently labelled secondary antibodies (Table 2) for $60 \mathrm{~min}$ at room temperature. The membranes were washed three times in PBS-T, and detected with a Li-cor scanner (Odyssey; Li-cor Biosciences, Lincoln, NE, USA).

\section{Luminex analysis}

After $24 \mathrm{~h}$ of co-culture, the secretome of HSCs was evaluated using the 9-plex kit, including tumor necrosis factor alpha (TNF $\alpha$ ), metalloproteinase 9 (MMP-9), metalloproteinase 2 (MMP-2), interferon gamma (IFN)$\gamma$, hepatocyte growth factor (HGF), interleukin 6 (IL-6), metalloproteinase 1 (MMP-1), interleukin 10 (IL-10), and metalloproteinase 13 (MMP-13) (R\&D Systems, Minneapolis, MN, USA) and Luminex technology (BioPlex 200; Bio-Rad Laboratories, Hercules, CA, USA). The supernatants were prepared as previously described for the pro-collagen type I C-Peptide ELISA. The assays were performed according to the manufacturer's instructions. The data were analyzed using Bio-Plex Manager 6.0 (Bio-Rad Laboratories).

\section{HGF neutralization}

HGF was neutralized with an anti-HGF antibody $(20 \mu \mathrm{g} /$ $\mathrm{mL} ; \mathrm{R} \& \mathrm{D}$ Systems) diluted in conditioned medium. The conditioned medium without the anti-HGF antibody was used as a control. The antibody was incubated in ADHLSC- or HSC-conditioned medium for $1 \mathrm{~h}$ at $37^{\circ} \mathrm{C}$ before adding to HSCs. After 24-h incubation, HSCs were harvested for counting, viability assays, and secretome analysis.

\section{Induction of liver fibrosis in young rats}

All animal experiments were performed in compliance with Belgian law for animal protection and were approved by the local ethics review board. All ADHLSCs used in the current study were cryopreserved at the fifth passage. On-site newborn Wistar-Han rats (Charles River, Lyon, France) were intraperitoneally injected with phenobarbital (30 mg $/ \mathrm{kg}^{-1} / \mathrm{day}^{-1}$ ) until day 4 . Then, phenobarbital was added to the drinking water $(0.5 \mathrm{~g} / \mathrm{L})$ until the end of the experiment. On postnatal day $8, \mathrm{CCl}_{4}$ (diluted in corn oil) 
was subcutaneously injected twice a week at increasing concentrations $(0.2-0.5 \mathrm{~mL} / \mathrm{kg})$ until 2 days post-cell transplantation.

\section{Cell transplantation}

At 3 weeks old, ADHLSCs $\left(12.5 \times 10^{6}\right.$ per $\mathrm{kg}$; viability $>90 \%$ by trypan blue exclusion assay) were intrahepatically transplanted into male rats once a week for 2 or 3 weeks under isoflurane (1.5\%) anesthesia. The rats were followed for 3 days post-transplantation. The animals were sacrificed on postnatal day 48 . Liver samples were recovered for mRNA and protein analyses $(n=6-$ 8 per group).

\section{Statistics}

Results are expressed as mean \pm standard error of the mean (SEM). Statistical differences were determined by paired Student's $t$ test for analysis of two samples and one-way ANOVA followed by the Newman-Keuls post hoc test. Differences were considered significant

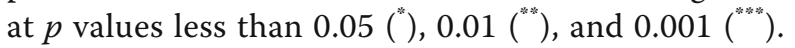

\section{Results \\ ADHLSCs inhibit both the adhesion and proliferation of HSCs}

To investigate the effects of ADHLSCs on activated HSCs, we used the Transwell co-culture system. Activated HSCs are characterized by an increased proliferation rate; therefore, we first analyzed the impact of co-culture on HSC yield. HSCs were plated at a fixed concentration of $1.0 \times 10^{4}$ cells $/ \mathrm{cm}^{2}$ in the lower chamber, and ADHLSCs were seeded on the collagencoated membrane insert at different densities to obtain ratios of $0: 1,1: 100,1: 10$, and 1:1. Activated HSCs (from four different donors) were collected after $24 \mathrm{~h}$, 4 days, and 7 days of co-culture. A significant decrease in the number of recovered HSCs was noted after $24 \mathrm{~h}$. The greatest decrease was observed at the lowest ADHLSC density (a 1:100 ratio; Fig. 1a, b). This inhibitory effect was maintained at 4 and 7 days (Fig. 1b). Comparative analysis of the proliferation index from day 1 to day 7 among all groups (0:1, 1:1, 1:10, and $1: 100)$ revealed that this coefficient was the same between all analyzed groups, which means that the inhibitory effect was initiated during the first $24 \mathrm{~h}$ of co-culture.

To confirm the counting data observed at $24 \mathrm{~h}$ of coculture, we used the CCK- 8 assay. Using spectrophotometry, we confirmed that HSCs co-cultured with ADHLSCs at a ratio of 1:100 showed a $30 \%$ decrease in cell number (Fig. 1c). Then, we further analyzed the mechanisms behind the reduced number of adhering HSCs obtained after $24 \mathrm{~h}$ of co-culture at a 1:100 ratio.
We investigated whether this could be related to poor adherence, inhibition of proliferation, and/or induced cell death. We first demonstrated that the decreased number of adherent HSCs was correlated with a significant, fourfold greater number of cells in the floating fraction in the culture supernatant (Additional file 1: Figure S1A).

We also checked whether the same effect could be observed with ADHLSC-conditioned medium. Both ADHLSCs and HSCs (as controls) were seeded separately, at the same initial density as was used in the co-cultures. Twenty-four hours later, the culture supernatants were collected and incubated with HSCs for $24 \mathrm{~h}$. The described effect was only observed when conditioned medium from ADHLSCs was used (Additional file 1: Figure S1B). We also observed that the inhibitory effect of ADHLSCs (or conditioned medium) on HSCs was maintained even when allogeneic ADHLSCs were used (data not shown). Next, we evaluated whether the ADHLSCs had an effect on the cell death of HSCs by using Annexin V-PI staining and flow cytometry. We did not observe any effect on cell death. The same observations were made for HSCs incubated with ADHLSCs and with conditioned medium. The analysis showed that the majority of the cells were viable (Additional file 1: Figure S1C). To understand the kinetics of this deficient adhesion, we analyzed the number of floating and adhering HSCs at different time points post-seeding in the presence of either ADHLSC- or HSC-conditioned medium. Our results demonstrated that early after seeding, in the presence of ADHLSC-conditioned medium the number of HSCs in the floating fraction is twice as high as the number in the presence of control HSC-conditioned medium (Additional file 2: Figure S2A). This delayed adhesion remained, and it reached a maximum $24 \mathrm{~h}$ post-seeding. Likewise, the number of adhering cells was lower when incubated with ADHLSC-conditioned medium (Additional file 2: Figure S2B).

Furthermore, we evaluated the effect of ADHLSCs on the cell cycle of HSCs by PI staining and flow cytometry. We demonstrated that a significantly higher number of HSCs co-cultured for $24 \mathrm{~h}$ with ADHLSCs at ratio of 1:100 were blocked in G0/G1 phase when compared to the number in control cultures without ADHLSCs (Fig. 2a). Concomitantly, we observed a significant decrease in the number of HSCs in G2/M phase following co-culture with ADHLSCs (Fig. 2a). The same result was obtained when HSCs were incubated with ADHLSC-conditioned medium (Fig. 2b). By using immunocytochemistry for Ki67, we confirmed a significant decrease in the number of immune-positive HSC nuclei (in four different microscopy fields per donor) after a 24-h incubation with ADHLSC-conditioned medium (Fig. 2c). 

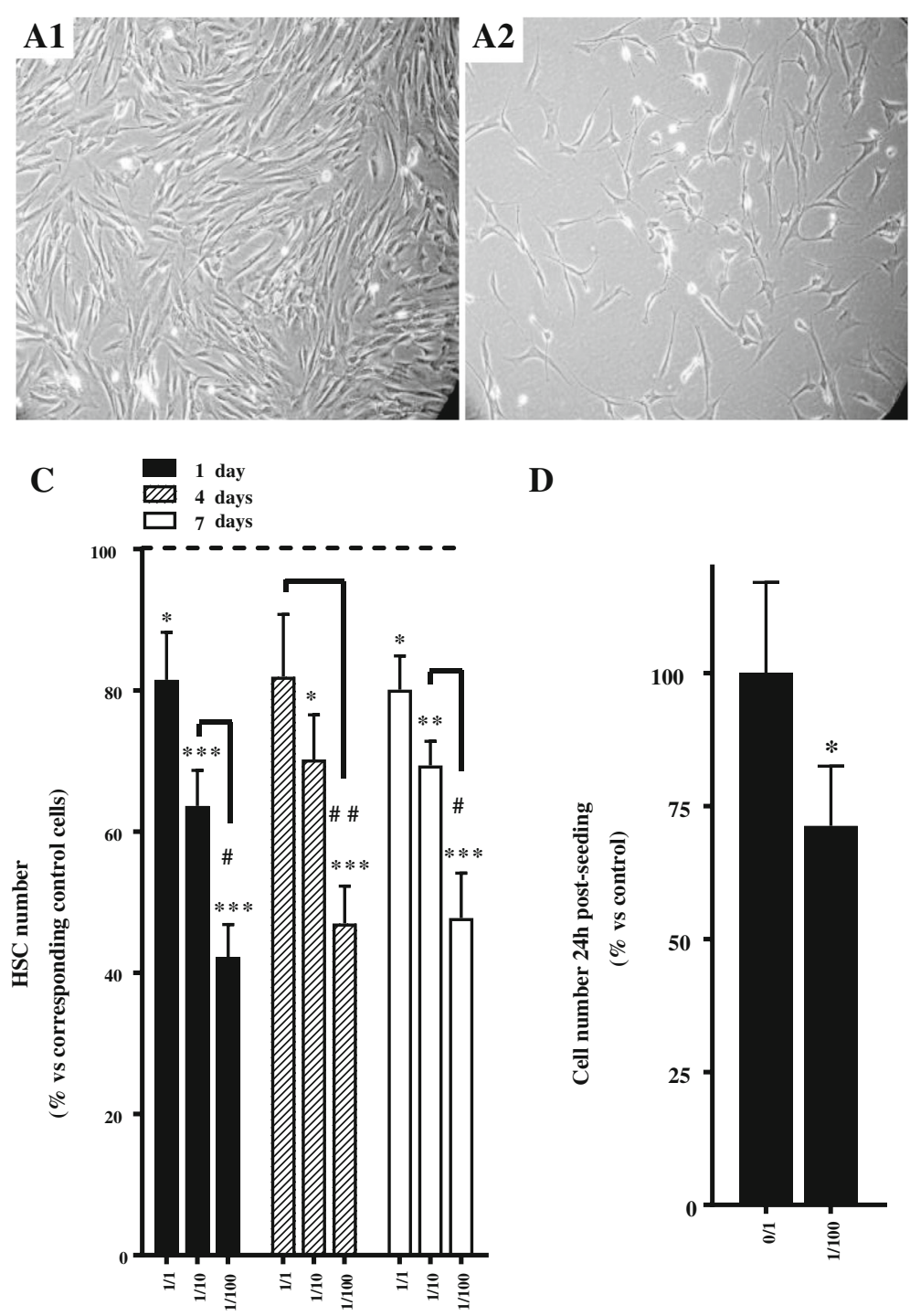

D

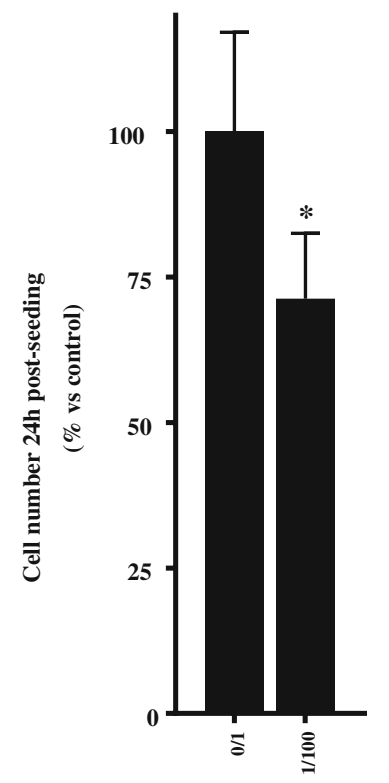

[Ratio ADHLSC/HSC]

Fig. 1 Effect of ADHLSCS on HSC culture. a Morphology of HSCs recovered after $24 \mathrm{~h}$ of co-culture without ADHLSCS (A1) or with ADHLSCS at an ADHLSC:HSC ratio of 1:100 (A2). b Significant decrease in the number of adherent HSCs after $24 \mathrm{~h}$ of co-culture at an ADHLSC:HSC ratio of 1:100, which was maintained for up to 7 days ( $\mathrm{N}=6$ for $24 \mathrm{~h} ; \mathrm{N}=4$ for 4 and 7 days). c Significant decrease in the number of HSCs after $24 \mathrm{~h}$ of co-culture at an ADHLSC:HSC ratio of 1:100, using the CCK-8 biochemical assay $(N=4)$. Results are expressed as mean \pm standard error of the mean (SEM). ${ }^{* * *} p$ value $<0.001 ;{ }^{* *} p<0.01 ;{ }^{*} p<0.05$. ADHLSCS adult-derived human liver mesenchymal stem/progenitor cells, HSC hepatic stellate cells

\section{ADHLSCs downregulate HSC secretion of pro-collagen} type I and stimulate the release of anti-fibrotic molecules Upon activation, the secretion of collagen type I by HSCs is increased. Therefore, we investigated the effect of ADHLSCs on the collagen secretion capacity of HSCs by measuring pro-collagen I (a precursor of collagen type I) secretion by ELISA. After $24 \mathrm{~h}$ of co-culture, we observed a significant (45\%) decrease in the amount of pro-collagen I secreted by HSCs when co-cultured with ADHLSCs (Fig. 3a). Using a multiplex Luminex assay, we also demonstrated that the level of HGF and IL- 6 secreted by HSCs was increased when co-cultured with ADHLSCs for $24 \mathrm{~h}$
(Fig. 3b). The secreted levels of the metalloproteinases MMP1 and MMP2 were also increased after 24 h of coculture with ADHLSCs (Table 3).

\section{Neutralization of HGF reverses the inhibitory effect of ADHLSCs on the number of HSCs and the secretion profile}

ADHLSCs secrete high levels of HGF, a growth factor known to have anti-fibrotic properties [17]. As the inhibitory effect of ADHLSCs on HSC proliferation seems to be mediated by soluble factors, we investigated the potential role of HGF by neutralizing this growth factor. 

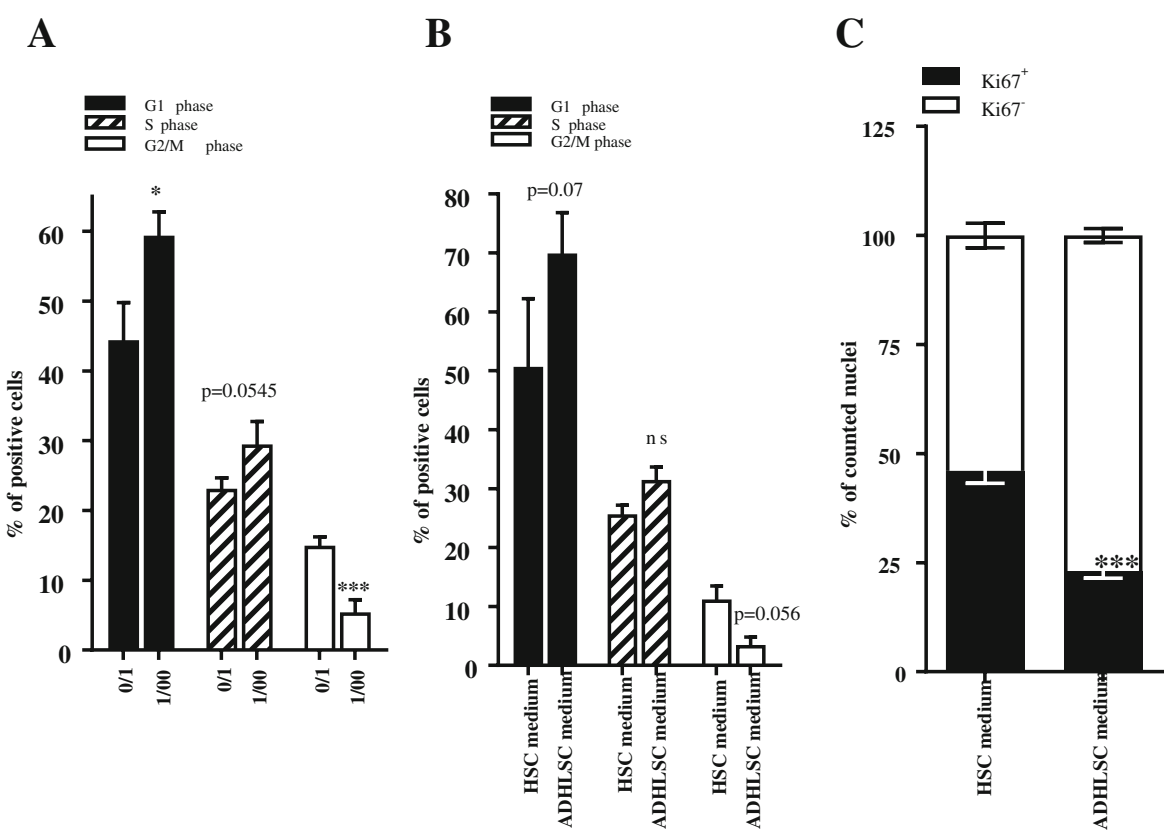

Fig. 2 Effect of ADHLSCS on HSC proliferation. Cell cycle analysis of Pl-stained HSCs demonstrated an increase in the number of HSC blocked in G0/G1 phase and a decrease in the number of HSCs in G2/M phase after $24 \mathrm{~h}$ of co-culture at an ADHLSC:HSC ratio of 1:100 (a) and after $24 \mathrm{~h}$ of incubation with ADHLSC-conditioned medium $(\mathbf{b})(\mathrm{N}=4)$. c The Ki67 immunostaining of HSCs showed a significant decrease in the number of immunostained nuclei of HSCs pre-incubated for $24 \mathrm{~h}$ with ADHLSC conditioned medium $(\mathrm{N}=4)$. For each experimental condition, four different fields were analyzed, and a total of 2500 nuclei were counted using Image J software. Results are expressed as mean \pm standard error of the mean (SEM). ${ }^{* * *} p$ value $<0.001 ;{ }^{* *} p<0.01 ;{ }^{*} p<0.05$. ADHLSCS adult-derived human liver mesenchymal stem/progenitor cells, HSC hepatic stellate cells

HSCs were incubated with conditioned medium that was pre-incubated with an anti-HGF antibody. After $24 \mathrm{~h}$, we did not observe any effect on the floating HSC population (Fig. 4a). Nevertheless, HGF appears to be more involved in modulating the fraction of adherent HSCs. Indeed, when the anti-HGF antibody was diluted in the ADHLSC-conditioned medium, it only partially inhibited the decrease in HSCs (Fig. 4a). We also demonstrated that the anti-HGF antibody blocked the inhibitory effect of ADHLSC-conditioned medium on the secretion of collagen, IL-6, HGF, MMP1, and MMP2 by HSCs (Fig. 4b, c).

\section{Transplantation of ADHLSCs into a juvenile rat model of} liver fibrosis decreases the expression of genes related to HSC activation

To assess whether ADHLSCs could modulate HSC activation in vivo, we transplanted MSCs transhepatically into young rats that were chronically treated with phenobarbital and $\mathrm{CCl}_{4}$ [28]. In the first group, ADHLSCs were transplanted twice (once per week) to reach a total number of $25 \times 10^{6}$ transplanted cells $/ \mathrm{kg}$ body weight, and analyses were performed 3 weeks later (the $\mathrm{CCl}_{4}$ treatment was maintained during this time period). In the second group, ADHLSCs were transplanted three times to reach a total number of $37.5 \times 10^{6}$ cells $/ \mathrm{kg}$ body weight, and the analyses were performed 2 weeks later. The expression of genes related to HSC activation/liver fibrosis was estimated by using RT-qPCR. Our data showed that the expression of $\alpha$-SMA, collagen $1 \alpha 1$, Loxl1, and TIMP- 1 was decreased in three out of six rats in the group that was transplanted twice compared to the levels in vehicletransplanted rats (Fig. 5a). In rats that were transplanted three times, the expression of such markers was decreased in three out of five rats (Fig. 5b). We also checked the protein expression of HSC activation markers, and observed that $\alpha$-SMA protein expression was significantly decreased in $\mathrm{CCl}_{4}$-treated, celltransplanted rats (in both groups) as compared to the expression in the $\mathrm{CCl}_{4}$-treated, nontransplanted group (Fig. 5c). The same observation was confirmed for another myofibroblastic marker fibronectin (Fig. 5c).We also showed that the rats displaying a decreased $\alpha$ SMA expression level after ADHLSC transplantation (either two or three times) also exhibited lower concentrations of liver transaminases (Additional file 3).

\section{Discussion}

In the current study, we demonstrated the inhibitory effect of ADHLSCs on HSC activation both in vitro and in vivo. In vitro, we showed that ADHLSCs inhibit the proliferation of HSCs, decrease their pro-collagen production, and induce the secretion of anti-fibrotic molecules. Such 

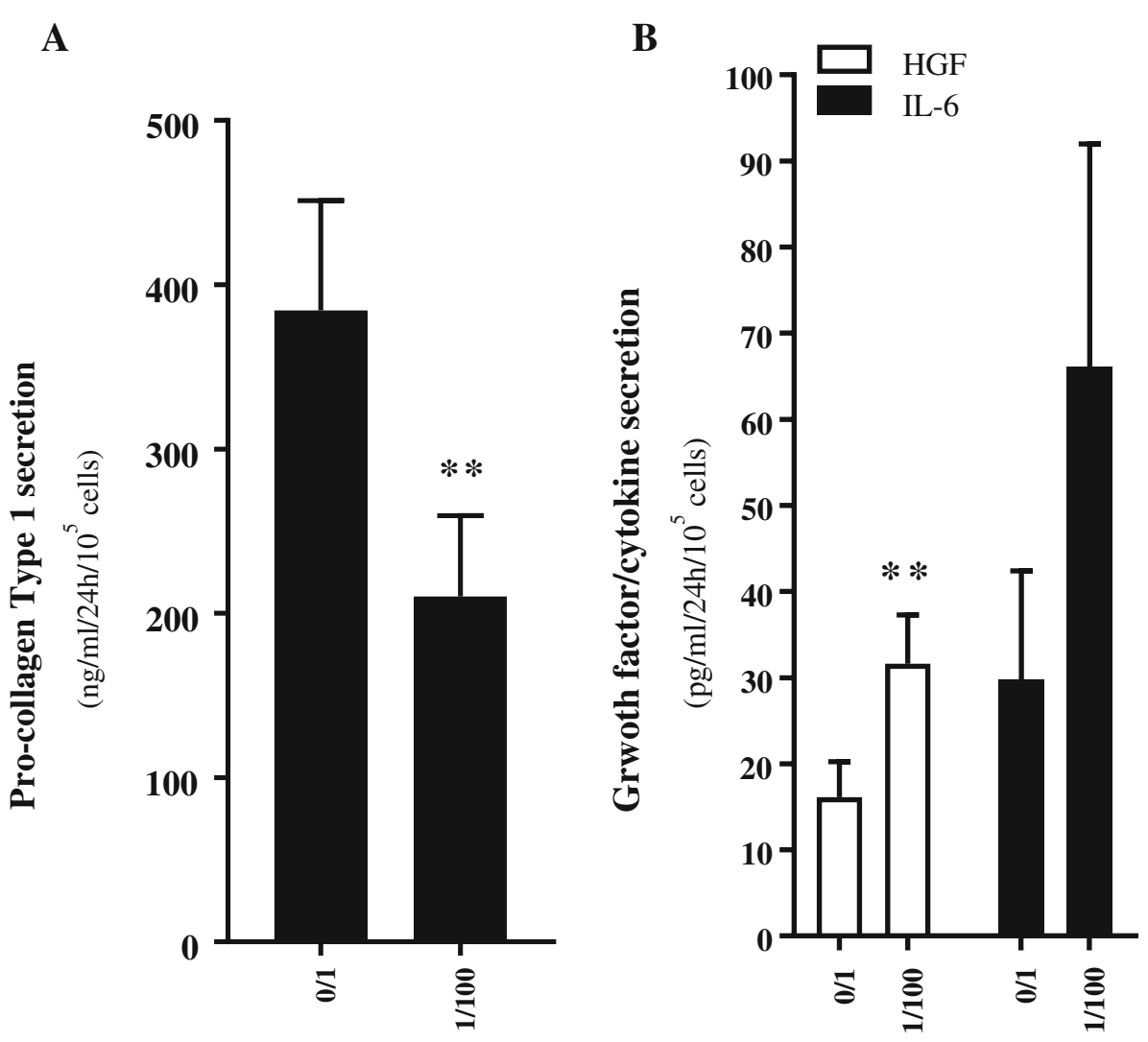

Fig. 3 Modulation of the HSC secretome after $24 \mathrm{~h}$ of co-culture with ADHLSCs. a ADHLSCs inhibit pro-collagen type I secretion by HSCs after $24 \mathrm{~h}$ of co-culture at a ADHLSC: HSC ratio of 1:100, as evaluated by ELISA ( $N=4)$. $\mathbf{b}$ The presence of ADHLSCs increased HGF and IL-6 secretion by HSCs after $24 \mathrm{~h}$ of co-culture at an ADHLSC:HSC ratio of 1:100, as evaluated by multiplex technology $(\mathrm{N}=4)$. Results are expressed as mean \pm standard error of the mean (SEM). ${ }^{* * *} p$ value $<0.001 ;{ }^{* *} p<0.01 ;{ }^{*} p<005$. HGF hepatocyte growth factor, IL-6 interleukin 6

effects are mediated via the HGF pathway. Our findings also showed, in an established rat model of liver fibrosis, a decrease in the expression of markers related to liver fibrosis following ADHLSC transplantation.

Because HSCs are the principal mediators of liver fibrosis, several therapeutic approaches have directly targeted their activation. However, the efficacy of the currently developed anti-fibrotic drugs has not been proven in humans $[1,6]$. MSC-based therapy for treating liver fibrosis based on the differentiation potential and immunoregulatory properties of MSCs is currently under evaluation [9, 29-31]. Cell-free mechanisms have also been proposed [31-33]. Our study investigated ADHLSC-HSC interactions with an in vitro co-culture system. Indeed, HSC activation is observed in vitro, as evidenced by the progressive loss of vitamin A stores, the increased proliferation rate, and extracellular matrix production when cultured on plastic [2]. In the co-culture system we used, we demonstrated that ADHLSCs could inhibit HSC proliferation after $24 \mathrm{~h}$ of co-culture through soluble factors constitutively secreted by ADHLSCs. The most potent effect was observed at an ADHLSC/HSC ratio of 1:100 and was reproduced with ADHLSC-conditioned medium (obtained $24 \mathrm{~h}$ after plating, at the same density used in the co-culture) and allogeneic ADHLSCs. The finding that the inhibition of HSC proliferation was inversely proportional to the number of ADHLSCs used is a striking difference compared to observations made with

Table 3 Levels of various metalloproteinases secreted by HSCs when co-cultured with or without ADHLSCs for $24 \mathrm{~h}$

\begin{tabular}{|c|c|c|c|c|c|c|c|c|}
\hline & \multicolumn{4}{|c|}{$\begin{array}{l}\text { MMP1 } \\
\left(\mathrm{pg} / \mathrm{mL}^{-1} / 24 \mathrm{~h}^{-1} / 10^{5} \text { cells }^{-1}\right)\end{array}$} & \multicolumn{4}{|c|}{$\begin{array}{l}\text { MMP2 } \\
\left(\mathrm{pg} / \mathrm{mL}^{-1} / 24 \mathrm{~h}^{-1} / 10^{5} \text { cells }^{-1}\right)\end{array}$} \\
\hline & Donor 1 & Donor 2 & Donor 3 & Donor 4 & Donor 1 & Donor 2 & Donor 3 & Donor 4 \\
\hline \multicolumn{9}{|c|}{ ADHLSC to HSC ratio } \\
\hline $0: 1$ & 474.18 & 1183.05 & 1005.03 & 4550.99 & 329.02 & 469.68 & 1919.75 & 1959.93 \\
\hline $1: 100$ & 843.57 & 1515.79 & 2248.78 & 6462.41 & 466.6 & 718.12 & 3288.16 & 2496.38 \\
\hline
\end{tabular}




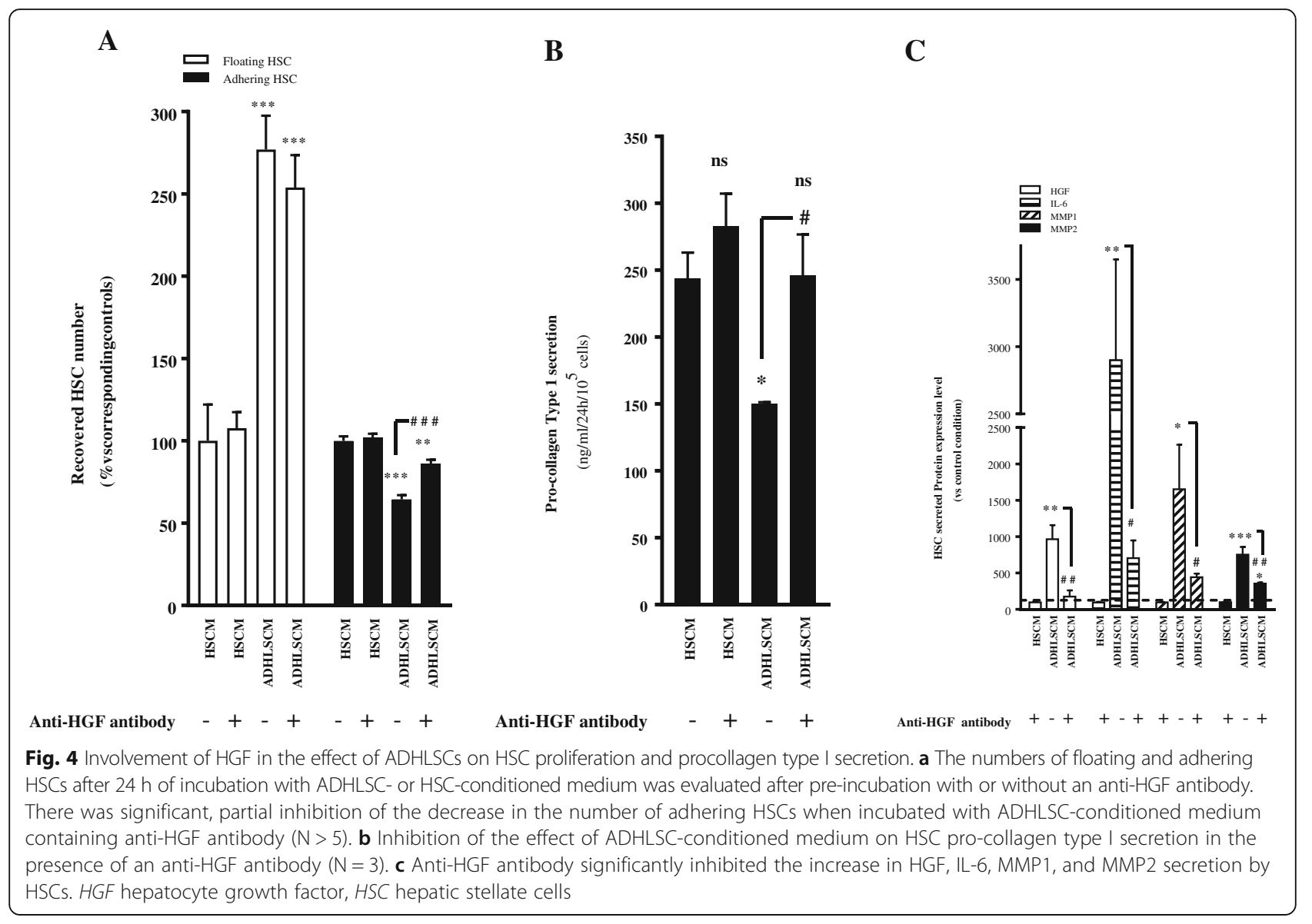

other extrahepatic MSCs [24, 25]. This difference could be explained in one of two ways; first, an inhibitory molecule secreted by ADHLSCs or the HSCs themselves when mixed at higher ADHLSC:HSC ratios, and second, more efficient action of the soluble anti-fibrotic factor(s) at a certain concentration. To further characterize the mechanism of HSC proliferation inhibition, we blocked the secretion of HGF, one of the factors that are highly secreted by ADHLSCs [17]. HGF neutralization partially reversed the inhibitory effect of ADHLSCs on HSC proliferation. These data are in accordance with other published data describing the ability of HGF to inhibit the plateletderived growth factor (PDGF)-mediated overproliferation of myofibroblasts [34].

The decrease in the amount of pro-collagen type 1 secreted by HSCs is promising, as the collagen type 1 is a major component of the extracellular matrix that is produced in excess during the development of liver fibrosis. HGF neutralization also reversed the ADHLSCinduced inhibition of HSC pro-collagen I secretion, in accordance with previous data showing the ability of HGF to inhibit the profibrogenic transforming growth factor beta (TGF- $\beta$ ) pathway [34]. HGF can induce the production of metalloproteinases by lung myofibroblasts
[34], and we showed that HSCs also increased the secretion of MMP1 and 2, which are both involved in extracellular matrix degradation. The increase in the secretion of IL- 6 by HSCs in our model could also be of therapeutic importance, as this cytokine has been implicated in the regeneration and survival of hepatocytes [35]. A recent study demonstrated that IL-6 and murine MSCs synergistically enhanced hepatic repair, improved hepatic function, and reduced liver fibrosis in mice [36].

Our in vivo findings showed that after ADHLSC transplantation, mRNA expression of genes related to fibrosis, including $\alpha$-SMA, Col1 $\alpha 1$, Loxl1, and TIMP-1 was decreased in three out of six rats that were infused twice (one infusion per week). This effect was observed 3 weeks after the last cell infusion. In the group of rats transplanted three times, the decreased mRNA expression of the above-mentioned genes was observed in three out of five animals 2 weeks after the last cell injection. Myofibroblast protein markers, including $\alpha-$ SMA and fibronectin which were reported to be upregulated after $\mathrm{CCl}_{4}$-induced liver fibrosis in rats [37, 38], were also significantly decreased after ADHLSC transplantation at least in half of the analyzed rats in each group. Although the data from the thrice-transplanted 


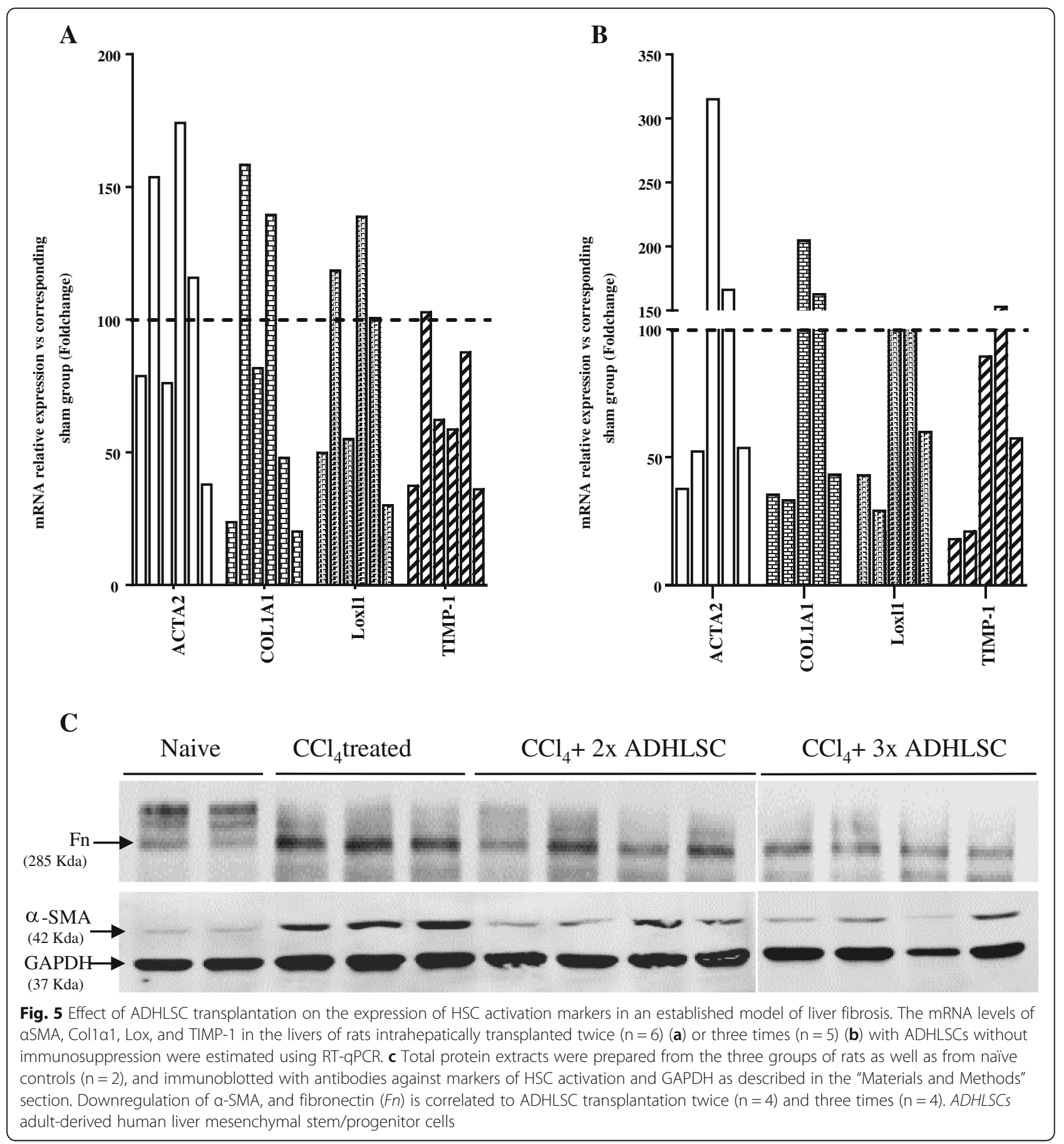

group seemed better, we cannot exclude the fact that the post-transplantation timing of the analysis may explain these potential differences. The group with two injections was analyzed 3 weeks after the last injection, whereas the group with three injections was analyzed only 2 weeks after the last injection.

Both groups were transplanted without immunosuppression, and no human cells were detected in the transplanted rat livers after targeting human $\mathrm{Ku}-80$ via immunohistochemistry (data not shown). These data were in accordance with a recent study claiming that MSCs are cleared in $\mathrm{CCl}_{4}$-damaged livers by 7 days posttransplantation [39] and support the cell-free mechanism of transplanted ADHLSCs in that liver fibrosis model.

\section{Conclusions}

The present study investigates the ability of ADHLSCs to modulate the activation of HSCs, a key process involved 
in liver fibrosis establishment. In summary, obtained data demonstrate that ADHLSCs inhibit HSC proliferation and pro-collagen secretion in vitro via paracrine mechanisms. Increased secretion of other anti-fibrotic factors by HSCs, like IL-6, was also noted upon coculture with ADHLSC. Those effects seem to be mediated at least in part, by the anti-fibrotic molecule HGF, highly secreted by ADHLSC. The study also shows that repeated intrahepatic transplantation of ADHLSCs in a rat model of liver fibrosis is correlated with a decrease in the expression of HSC activation markers. Taken together, our data support the further development of ADHLSCs and/or their secretome for the treatment of liver fibrosis.

\section{Additional files}

Additional file 1: Figure S1. Effect of ADHLSC on HSC plating efficiency. (A) The counting of floating and adhering HSC number after 24 hours of co-culture with ADHLSC showed an increase in the number of floating $\mathrm{HSC}$ concomitant with a decrease in the number of adhering HSC at an ADHLSC/HSC ratio of 1/100 ( $n=4)$. (B) The counting of the number of floating and adhering HSC after 24 hours of incubation with ADHLSC conditioned medium showed similarly an increase in the $\%$ of floating $\mathrm{HSC}$ and a decrease in the \% of adhering $\mathrm{HSC}$, as compared with HSC incubated the same period of time with HSC conditioned medium $(n=4)$. C, Following annexin V-PI staining, no significant difference in cell death induction was noticed between HSC cultivated for 24 hours with ADHLSC or with HSC conditioned medium $(n=4)$. Results are expressed as mean \pm standard error of the mean (SEM). ${ }^{* * *}$ Denotes a $p$ value $<0.001$; ${ }^{* *} p<0.01 ;{ }^{*} p<0.05$. (PPT $271 \mathrm{~kb}$ )

Additional file 2: Figure S2. ADHLSC delay HSC post-seeding adhesion. The plating kinetic analysis revealed a higher number of floating HSC (A) and a lower number of adhering HSC (B) at 2, 4, 8 and 24 hours post-seeding in the group of HSC incubated with ADHLSC conditioned medium in comparison with the group incubated with HSC conditioned medium $(n=4)$. Results are expressed as mean \pm standard error of the mean (SEM). ${ }^{* * *}$ Denotes a $p$ value $<0.001 ;{ }^{* *} p<0.01 ;{ }^{*} p<0.05$. (PPT $205 \mathrm{~kb}$ )

Additional file 3: Collection of blood samples and measurement of liver enzymes. (DOCX 18 kb)

\section{Abbreviations}

ADHLSCs: Adult-derived human liver mesenchymal stem/progenitor cells; DMEM: Dulbecco's modified Eagle's medium; HGF: Hepatocyte growth factor; HSC: Hepatic stellate cells; IL-6: Interleukin 6; MMP1: Metalloproteinase 1, MMP2, Metalloproteinase 2; MSC: Mesenchymal stem cells

\section{Acknowledgements}

Silvia Berardis was supported by the Université Catholique de Louvain, Fonds Spécial de Recherche (FSR) and by the Fonds National de la Recherche Scientifique (FNRS). Hoda El-Kehdy is the holder of a post-doc grant from Institut de Recherche Experimentale et Clinique (IREC). Mustapha Najimi is the Principal Investigator of IREC.

\section{Funding}

This work was financially supported by Région Bruxelloise, Innoviris (BruStem project).

\section{Availability of data and materials}

All data generated or analyzed during this study are included in this published article (and its Additional files)

\section{Authors' contributions}

$\mathrm{MN}$ conceived and designed the experiments, analyzed the data, and wrote and reviewed the manuscript. ES conceived and designed the experiments, analyzed the data, and wrote and reviewed the manuscript. SB performed the experiments, analyzed the data, and wrote and reviewed the manuscript. VR performed the experiments. JE performed the experiments. HEK performed the experiments. $\mathrm{CL}$ analyzed the data. PH analyzed the data, and wrote and reviewed the manuscript. AET contributed material tools. LVG contributed material tools, and wrote and reviewed the manuscript. All authors read and approved the final manuscript.

\section{Competing interests}

The authors declare that they have no competing interests.

\section{Consent for publication}

Not applicable.

\section{Ethics approval and consent to participate}

The study was approved by the institution ethics committee (Comité d'éthique hospitalo-facultaire, reference: JMM/sy/2010/12). All donors signed an informed consent form. Liver tissue was obtained from the Ministry of Health-approved hospital tissue bank.

\section{Publisher's Note}

Springer Nature remains neutral with regard to jurisdictional claims in published maps and institutional affiliations.

\section{Author details}

${ }^{1}$ Université Catholique de Louvain, Institut de Recherche Expérimentale et Clinique (IREC), Laboratory of Pediatric Hepatology and Cell Therapy, Avenue Mounier, 52, 1200 Brussels, Belgium. ${ }^{2}$ Liver Cell Biology Lab, Vrije Universiteit Brussel (VUB), Laarbeeklaan 103, 1090 Brussels, Belgium. ${ }^{3}$ Cell Biology Unit, de Duve Institute, Université Catholique de Louvain, Avenue Hippocrate 75, 1200 Brussels, Belgium.

Received: 21 December 2016 Revised: 3 May 2017

Accepted: 5 May 2017 Published online: 05 June 2017

\section{References}

1. Friedman SL. Liver fibrosis - from bench to bedside. J Hepatol. 2003;38 Suppl 1:S38-53.

2. Friedman SL. Hepatic fibrosis - overview. Toxicology. 2008;254(3):120-9.

3. Francoz C, Belghiti J, Durand F. Indications of liver transplantation in patients with complications of cirrhosis. Best Pract Res Clin Gastroenterol. 2007;21(1):175-90

4. Evans HM, Kelly DA, McKiernan PJ, Hubscher S. Progressive histological damage in liver allografts following pediatric liver transplantation. Hepatology. 2006:43(5):1109-17.

5. Li JT, Liao ZX, Ping J, Xu D, Wang H. Molecular mechanism of hepatic stellate cell activation and anti-fibrotic therapeutic strategies. J Gastroenterol. 2008;43(6):419-28.

6. Weiskirchen R. Hepatoprotective and anti-fibrotic agents: it's time to take the next step. Front Pharmacol. 2016;6:303.

7. Friedman SL. Molecular regulation of hepatic fibrosis, an integrated cellular response to tissue injury. J Biol Chem. 2000;275(4):2247-50.

8. Friedman SL. Hepatic stellate cells: protean, multifunctional, and enigmatic cells of the liver. Physiol Rev. 2008;88(1):125-72.

9. Mormone E, George J, Nieto N. Molecular pathogenesis of hepatic fibrosis and current therapeutic approaches. Chem Biol Interact. 2011;193(3):225-31

10. Ren G, Chen X, Dong F, Li W, Ren X, Zhang Y, et al. Concise review: mesenchymal stem cells and translational medicine: emerging issues. Stem Cells Transl Med. 2012;1(1):51-8.

11. Sokal EM, Stephenne X, Ottolenghi C, Jazouli N, Clapuyt P, Lacaille F, et al. Liver engraftment and repopulation by in vitro expanded adult derived human liver stem cells in a child with ornithine carbamoyltransferase deficiency. JIMD Rep. 2014;13:65-72.

12. Sokal EM. Treating inborn errors of liver metabolism with stem cells: current clinical development. J Inherit Metab Dis. 2014;37(4):535-9. 
13. Khuu DN, Nyabi O, Maerckx C, Sokal E, Najimi M. Adult human liver mesenchymal stem/progenitor cells participate in mouse liver regeneration after hepatectomy. Cell Transplant. 2013;22(8):1369-80.

14. Najimi M, Khuu DN, Lysy PA, Jazouli N, Abarca J, Sempoux C, et al. Adultderived human liver mesenchymal-like cells as a potential progenitor reservoir of hepatocytes? Cell Transplant. 2007;16(7):717-28.

15. Khuu DN, Scheers I, Ehnert S, Jazouli N, Nyabi O, Buc-Calderon P, et al. In vitro differentiated adult human liver progenitor cells display mature hepatic metabolic functions: a potential tool for in vitro pharmacotoxicological testing. Cell Transplant. 2011;20(2):287-302.

16. Sana G, Lombard C, Vosters O, Jazouli N, Andre F, Stephenne X, et al. Adult human hepatocytes promote CD4+ T cell hyporesponsiveness via interleukin-10 producing allogeneic dendritic cells. Cell Transplant. 2014; 23(9):1127-42.

17. Berardis S, Lombard C, Evraerts J, El TA, Rosseels V, Sancho-Bru P, et al. Gene expression profiling and secretome analysis differentiate adult-derived human liver stem/progenitor cells and human hepatic stellate cells. PLoS One. 2014;9(1):e86137.

18. Le BK, Ringden O. Immunomodulation by mesenchymal stem cells and clinical experience. J Intern Med. 2007;262(5):509-25.

19. Ortiz LA, Dutreil M, Fattman C, Pandey AC, Torres G, Go K, et al. Interleukin 1 receptor antagonist mediates the anti-inflammatory and anti-fibrotic effect of mesenchymal stem cells during lung injury. Proc Natl Acad Sci U S A. 2007;104(26):11002-7.

20. Defresne F, Tondreau T, Stephenne X, Smets F, Bourgois A, Najimi M, et al. Biodistribution of adult derived human liver stem cells following intraportal infusion in a 17-year-old patient with glycogenosis type 1A. Nucl Med Biol. 2014:41(4):371-5.

21. Banas A, Teratani T, Yamamoto Y, Tokuhara M, Takeshita F, Osaki M, et al. IFATS collection: in vivo therapeutic potential of human adipose tissue mesenchymal stem cells after transplantation into mice with liver injury. Stem Cells. 2008;26(10):2705-12.

22. Tsai PC, Fu TW, Chen YM, Ko TL, Chen TH, Shih YH, et al. The therapeutic potential of human umbilical mesenchymal stem cells from Wharton's jelly in the treatment of rat liver fibrosis. Liver Transpl. 2009;15(5):484-95.

23. Parekkadan B, Van PD, Megeed Z, Kobayashi N, Tilles AW, Berthiaume F, et al. Immunomodulation of activated hepatic stellate cells by mesenchymal stem cells. Biochem Biophys Res Commun. 2007:363(2):247-52.

24. Wang J, Bian C, Liao L, Zhu Y, Li J, Zeng L, et al. Inhibition of hepatic stellate cells proliferation by mesenchymal stem cells and the possible mechanisms. Hepatol Res. 2009;39(12):1219-28.

25. Lin N, Hu K, Chen S, Xie S, Tang Z, Lin J, et al. Nerve growth factormediated paracrine regulation of hepatic stellate cells by multipotent mesenchymal stromal cells. Life Sci. 2009;85(7-8):291-5.

26. El Taghdouini A, Najimi M, Sancho-Bru P, Sokal E, van Grunsven LA. In vitro reversion of activated primary human hepatic stellate cells. Fibrogenesis Tissue Repair. 2015;8:14.

27. El-Kehdy H, Sargiacomo C, Fayyad-Kazan M, Fayyad-Kazan H, Lombard C, Lagneaux L, et al. Immunoprofiling of adult-derived human liver stem/ progenitor cells: impact of hepatogenic differentiation and inflammation. Stem Cells Int. In press

28. Sokal EM, Mostin J, Buts JP. Liver metabolic zonation in rat biliary cirrhosis: distribution is reverse of that in toxic cirrhosis. Hepatology. 1992;15(5):904-8

29. Najimi M, Defresne F, Sokal EM. Concise review: updated advances and current challenges in cell therapy for inborn liver metabolic defects. Stem Cells Transl Med. 2016;5(8):1117-25.

30. Jung $\mathrm{KH}$, Shin HP, Lee $\mathrm{S}$, Lim YJ, Hwang SH, Han H, et al. Effect of human umbilical cord blood derived mesenchymal stem cells in a cirrhotic rat model. Liver Int. 2009;29(6):898-909.

31. Chang YJ, Liu JW, Lin PC, Sun LY, Peng CW, Luo GH, et al. Mesenchymal stem cells facilitate recovery from chemically induced liver damage and decrease liver fibrosis. Life Sci. 2009:85(13-14):517-25.

32. Rabani V, Shahsavani M, Gharavi M, Piryaei A, Azhdari Z, Baharvand $H$. Mesenchymal stem cell infusion therapy in a carbon tetrachloride-induced liver fibrosis model affects matrix metalloproteinase expression. Cell Biol Int. 2010;34(6):601-5.

33. Tanimoto $H$, Terai $S$, Taro T, Murata $Y$, Fujisawa $K$, Yamamoto $N$, et al. Improvement of liver fibrosis by infusion of cultured cells derived from human bone marrow. Cell Tissue Res. 2013;354(3):717-28.
34. Nakamura T, Mizuno S. The discovery of hepatocyte growth factor (HGF) and its significance for cell biology, life sciences and clinical medicine. Proc Jpn Acad Ser B Phys Biol Sci. 2010;86(6):588-610.

35. Kovalovich K, Li W, DeAngelis R, Greenbaum LE, Ciliberto G, Taub R. Interleukin-6 protects against Fas-mediated death by establishing a critical level of anti-apoptotic hepatic proteins FLIP, BCl-2, and BCl-xL. J Biol Chem. 2001;276(28):26605-13.

36. Nasir GA, Mohsin S, Khan M, Shams S, Ali G, Khan SN, et al. Mesenchymal stem cells and Interleukin-6 attenuate liver fibrosis in mice. J Transl Med. 2013;11:78.

37. Liu XY, Liu RX, Hou F, Cui LJ, Li CY, Chi C, et al. Fibronectin expression is critical for liver fibrogenesis in vivo and in vitro. Mol Med Rep. 2016;14(4): 3669-75.

38. Wang S, Lee JS, Hyun J, Kim J, Kim SU, Cha HJ, et al. Tumor necrosis factorinducible gene 6 promotes liver regeneration in mice with acute liver injury. Stem Cell Res Ther. 2015;6:20

39. Park M, Kim YH, Woo SY, Lee HJ, Yu Y, Kim HS, et al. Tonsil-derived mesenchymal stem cells ameliorate CCl4-induced liver fibrosis in mice via autophagy activation. Sci Rep. 2015;5:8616.

\section{Submit your next manuscript to BioMed Central and we will help you at every step:}

- We accept pre-submission inquiries

- Our selector tool helps you to find the most relevant journal

- We provide round the clock customer support

- Convenient online submission

- Thorough peer review

- Inclusion in PubMed and all major indexing services

- Maximum visibility for your research

Submit your manuscript at www.biomedcentral.com/submit 